\title{
Sub-Rouse Dynamics in Poly(isobutylene) as a Function of Molar Mass
}

\author{
Marianna Spyridakou, ${ }^{1}$ Tanmoy Maji, ${ }^{2}$ Manos Gkikas, ${ }^{2}$ Kia L. Ngai, ${ }^{3}$ \\ George Floudas ${ }^{1,4^{*}}$ \\ ${ }^{1}$ Department of Physics, University of Ioannina, 45110 Ioannina, Greece \\ ${ }^{2}$ Department of Chemistry, University of Massachusetts Lowell, Cambridge, \\ Massachusetts, Lowell MA 01854, USA \\ ${ }^{3}$ CNR-IPCF, Università di Pisa, Largo Bruno Pontecorvo 3, I-56127, Pisa, Italy \\ ${ }^{4}$ University Research Center of Ioannina (URCI) - Institute of Materials Science and \\ Computing, 45110 Ioannina, Greece
}


Initiator Synthesis
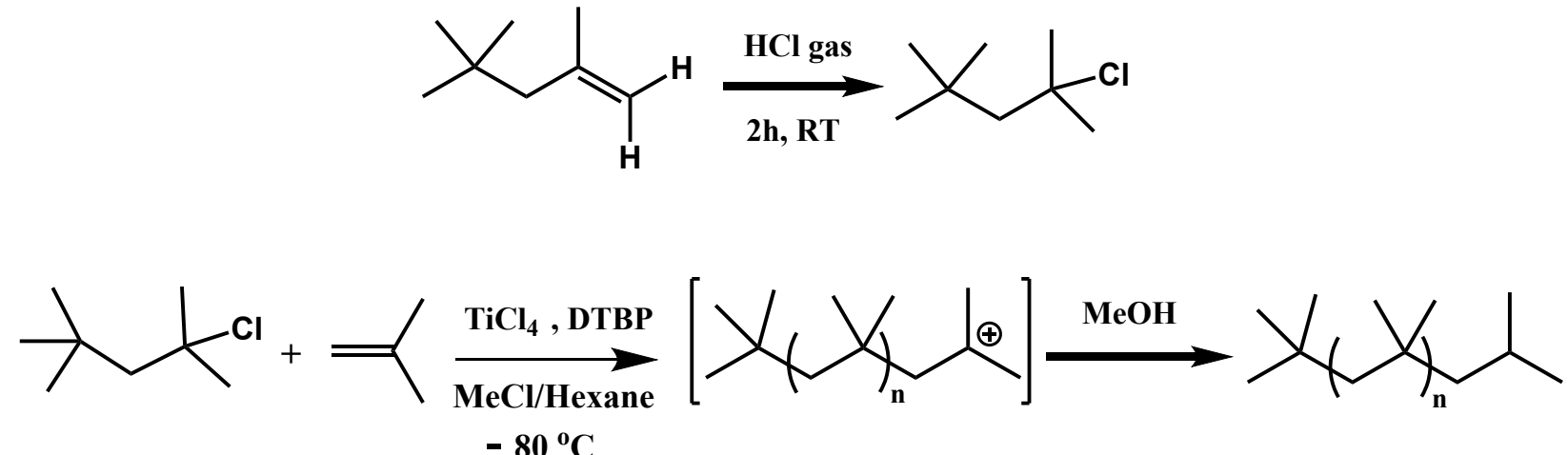

Scheme S1. Synthesis of initiator 2-chloro-2,4,4-trimethylpentane and formation of poly(isobutylenes) via living cationic polymerization.

(A)<smiles>CC=CCC(C)(C)C</smiles>

a

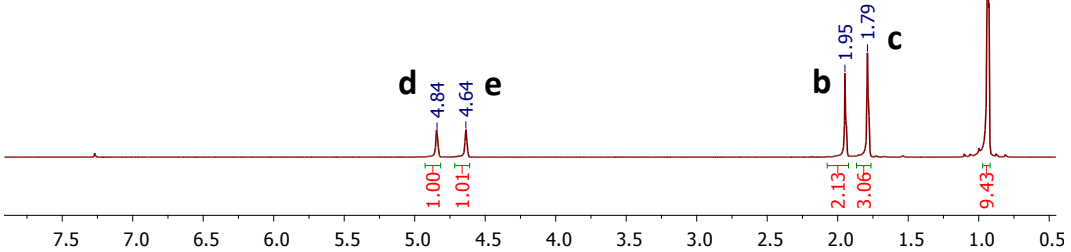

(B)

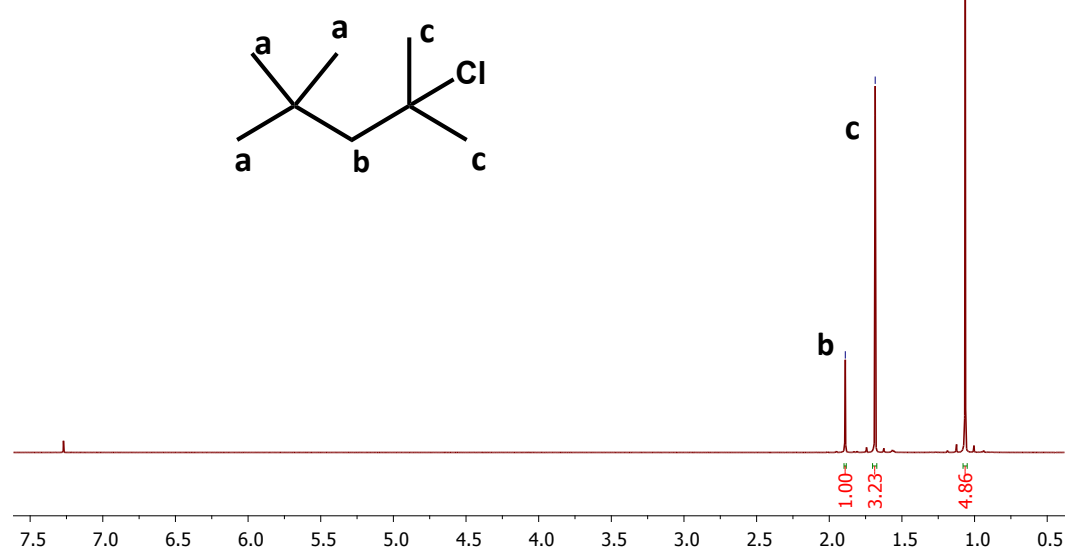

Figure S1. ${ }^{1} \mathrm{H}-\mathrm{NMR}$ spectra of (a) 2,4,4-trimethyl-1-pentene and (b) 2-chloro-2,4,4trimethyl-pentene in $\mathrm{CDCl}_{3}$. 